\title{
Nonsense surveillance regulates expression of diverse classes of mammalian transcripts and mutes genomic noise
}

\author{
Joshua T Mendell ${ }^{1}$, Neda A Sharifi ${ }^{1}$, Jennifer L Meyers ${ }^{2}$, Francisco Martinez-Murillo ${ }^{3}$ \& Harry C Dietz ${ }^{1,2}$
}

Premature termination codons induce rapid transcript degradation in eukaryotic cells through nonsense-mediated mRNA decay (NMD) ${ }^{1,2}$. This pathway can modulate phenotypes arising from nonsense or frameshift mutations, but little is known about the physiologic role of NMD in higher eukaryotes. To address this issue, we examined expression profiles in mammalian cells depleted of Rent1 (also called hUpf1), a factor essential for $\mathrm{NMD}^{3,4}$. Upregulated transcripts included those with upstream open reading frames in the $5^{\prime}$ untranslated region, alternative splicing that introduces nonsense codons or frameshifts, introns in the $3^{\prime}$ untranslated region or selenocysteine codons. Transcripts derived from ancient transposons and endogenous retroviruses were also upregulated. These RNAs are unified by the presence of a spliced intron at least 50 nucleotides downstream of a termination codon, a context sufficient to initiate $\mathrm{NMD}^{5}$.
Consistent with direct regulation by NMD, representative upregulated transcripts decayed more slowly in cells deficient in NMD. In addition, inhibition of NMD induced by amino acid starvation upregulated transcripts that promote amino acid homeostasis. These results document that nonsense surveillance is a crucial post-transcriptional regulatory event that influences the expression of broad classes of physiologic transcripts, has been functionally incorporated into essential homeostatic mechanisms and suppresses expression of evolutionary remnants.

The NMD pathway is conserved in all eukaryotic organisms that have been studied ${ }^{1,2}$. Strict evolutionary maintenance of this function may result from its ability to protect cells from deleterious truncated proteins that would be produced from stable nonsense transcripts. There are many well-studied examples of human

Table 1 Classes and selected examples of putative NMD-regulated transcripts

Putative NMD-inducing feature

\section{UORF}

PTC introduced by alternative splicing

Transposon or retrovirus
Gene

Relative increase (microarray value)

$\begin{array}{lr}\text { Mitogen-activated protein kinase kinase kinase 14 (MAP3K14) } & 11.3 \\ \text { Dual specificity phosphatase 10 (DUSP1O) } & 5.7 \\ \text { Asparagine synthetase (ASNS) } & 4.7 \\ \text { Interferon-related developmental regulator 1 (IFRD1) } & 3.5 \\ \text { PDZ domain containing 3 (PDZK3) } & 2.6 \\ \text { Activating transcription factor 3 (ATF3) } & 9.6 \\ \text { Uridine phosphorylase (UPP1) } & 6.3 \\ \text { Ras association (RalGDS/AF-6) domain family 1 (RASSF1) } & 4.1 \\ \text { Hypothetical protein FLJ11021 similar to splicing factor, arginine/serine-rich 4 } & 2.2 \\ \text { Hypothetical protein LOC162427 } & 2.1 \\ \text { Growth arrest and DNA-damage-inducible, beta (GADD45B) } & 6.2 \\ \text { Hypothetical protein DJ167A19.1 } & 3.4 \\ \text { Mitochondrial ribosomal protein L49 (MRPL49) } & 2.1 \\ \text { Dexamethasone-induced transcript (DEXI) } & 2.1 \\ \text { Thioredoxin reductase 2 ( } \text { (XNRD2) } & 7.2 \\ \text { Selenoprotein W, 1 (SEPW1) } & 1.9 \\ \text { Defective mariner transposon (HSMAR2) } & 7.1 \\ \text { Human endogenous retrovirus H (HERV-H) } & 5.9\end{array}$

${ }^{1}$ Institute of Genetic Medicine and ${ }^{2}$ Howard Hughes Medical Institute, Johns Hopkins University School of Medicine, 539 Broadway Research Building, 733 N. Broadway, Baltimore, Maryland, 21205, USA. ${ }^{3}$ Department of Molecular Biology and Genetics, Johns Hopkins University School of Medicine, 808 PCTB, 725 North Wolfe Street, Baltimore, Maryland, 21205, USA. Correspondence should be addressed to H.C.D. (hdietz@jhmi.edu). 


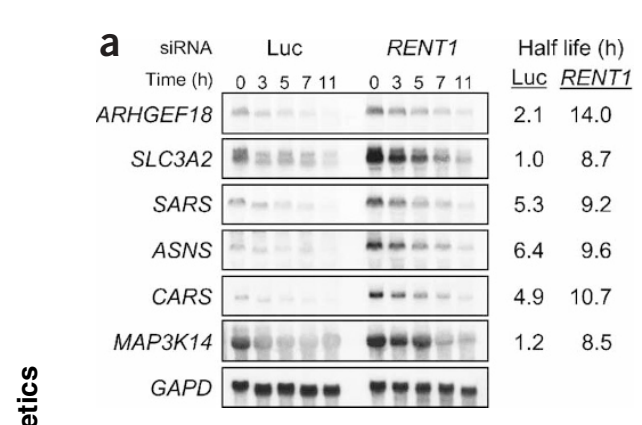

C

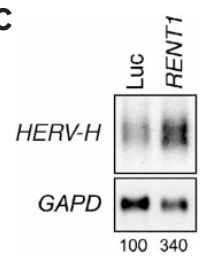

d

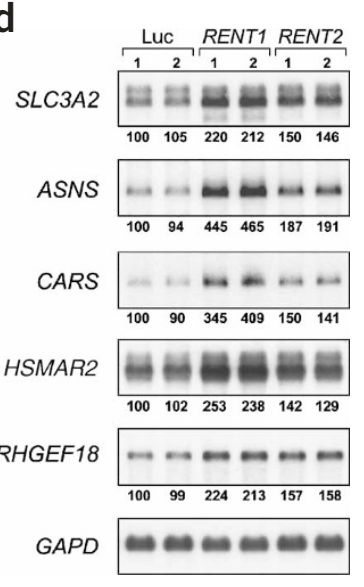

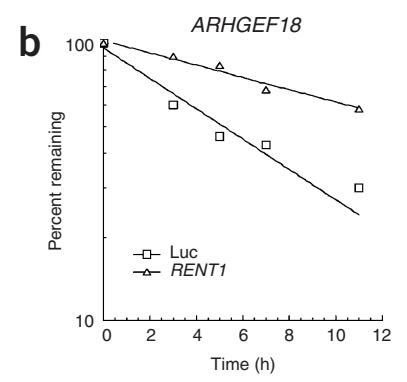
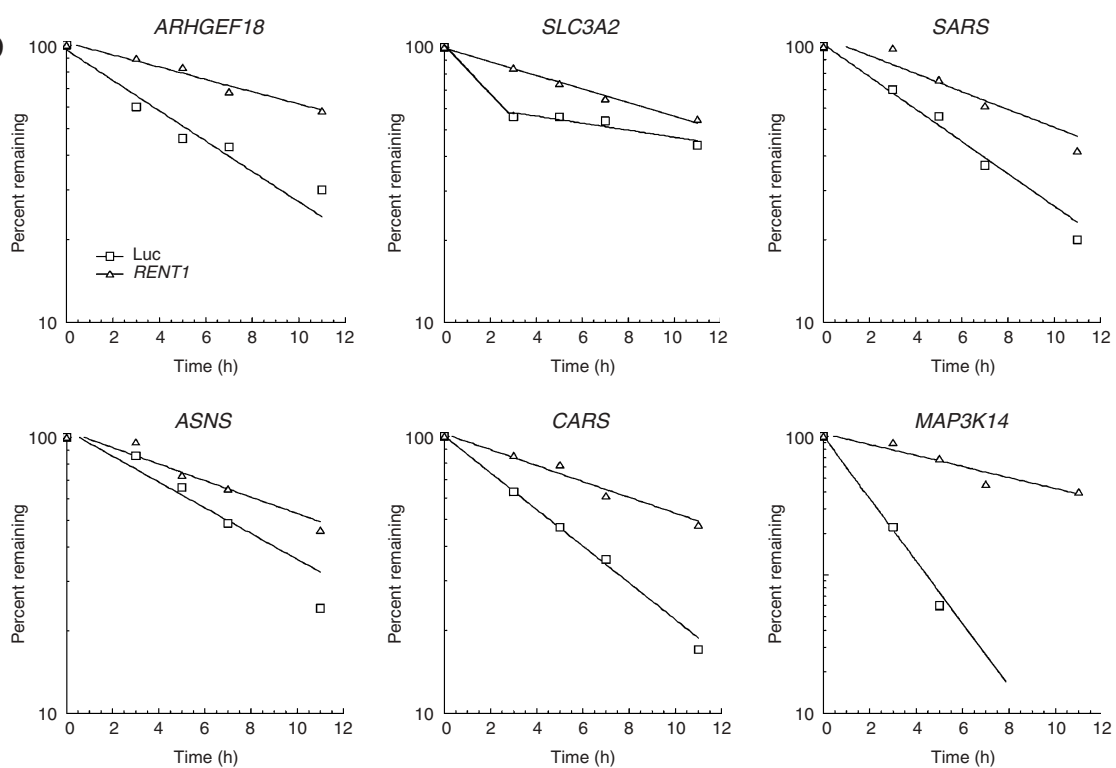

Figure 1 Prolonged decay rates and increased steady-state abundance of transcripts identified through expression profiling of Rent1-depleted cells. (a) Half-lives of selected upregulated transcripts. Seventytwo hours after treatment with RNA interference directed against firefly luciferase (Luc) or RENT1, transcription was inhibited with 5,6-dichloro-1- $\beta$-D-ribofuranosylbenzimidazole. RNA was collected at the indicated time points and transcript levels were monitored by northern blotting. Levels of GAPD, a stable housekeeping transcript, were used to correct for differences in RNA loading. (b) Northern blots shown in a were quantified, and values are represented as semi-log plots. All transcripts showed exponential decay except SLC3A2, which showed a biphasic decay pattern consistent with the behavior of other known nonsense transcripts ${ }^{30}$. (c) Northern-blot analysis of transcripts derived from endogenous retroviral sequences $(H E R V-H)$ in Rent1-depleted cells. (d) Steady-state abundance of selected upregulated transcripts in Rent1-depleted and Rent2-depleted cells. Two independent experiments are shown for each experimental condition (1 and 2). To quantify each transcript (normalized to GAPD), one luciferase siRNA-treated sample was arbitrarily set to 100 and other signals were adjusted relative to this value.

phenotypes resulting from nonsense or frameshift mutations that are modulated by $\mathrm{NMD}^{6,7}$. It seems unlikely, however, that the rare frequency of de novo nonsense mutations could provide sufficient selective pressure for complete evolutionary maintenance of NMD. Another view posits that physiologic transcripts that structurally mimic nonsense transcripts are the predominant substrates for NMD. Observations in model systems support this hypothesis. For example, expression profiling of NMD-deficient yeast strains found altered expression of $\sim 10 \%$ of the transcriptome, allowing identification of specific classes of physiologic NMD substrates ${ }^{8,9}$. In mammals,

Table 2 Half-lives of putative NMD-regulated transcripts

\begin{tabular}{|c|c|c|c|c|}
\hline Gene & $\begin{array}{l}\text { Putative NMD-inducing } \\
\text { feature }\end{array}$ & $\begin{array}{l}\text { Half-life Luc } \\
\text { siRNA (h) }\end{array}$ & $\begin{array}{c}\text { Half-life } \\
\text { Rent1 siRNA (h) }\end{array}$ & $\begin{array}{l}\text { Half-life } \\
\text { relative increase }\end{array}$ \\
\hline Mitogen-activated protein kinase kinase kinase 14 (MAP3K14) & uORFs & 1.2 & 8.5 & 7.1 \\
\hline Rho-specific guanine nucleotide exchange factor p114 (ARHGEF18) & uORF & 2.1 & 14.0 & 6.7 \\
\hline Protein tyrosine phosphatase, receptor type, $\mathrm{F}$ (PTPRF) & uORF & 3.8 & 24.9 & 6.6 \\
\hline Phosphatidylserine decarboxylase (PISD) & uORF & 3.8 & 8.0 & 2.1 \\
\hline Asparagine synthetase (ASNS) & uORFs & 6.4 & 9.6 & 1.5 \\
\hline Syntaxin $3 A(S T X 3 A)$ & uORFs & 4.1 & 6.2 & 1.5 \\
\hline DNAJ homolog B2 (DNAJB2) & 3' UTR intron & 2.3 & 8.3 & 3.6 \\
\hline Dibasic/neutral amino acid transporter (SLC3A2) & Alt. exon with PTC, uORF & 1.0 & 8.7 & 8.7 \\
\hline Activating transcription factor 3 (ATF3) & Alt. exon with PTC & 2.9 & 5.4 & 1.9 \\
\hline Uridine phosphorylase (UPP1) & Alt. splicing creates frameshift & 7.2 & 31.3 & 4.3 \\
\hline Selenoprotein W1 (SEPW1) & Selenoprotein & 9.3 & 15.3 & 1.6 \\
\hline Solute carrier family $25 A 14$ (SLC25A14) & Unknown & 7.5 & 15.8 & 2.1 \\
\hline Cysteinyl-tRNA synthetase (CARS) & Unknown & 4.9 & 10.7 & 2.2 \\
\hline Seryl-tRNA synthetase (SARS) & Unknown & 5.3 & 9.2 & 1.7 \\
\hline
\end{tabular}


Table 3 Upregulated human transcripts with amino acid metabolism and transport functions

\begin{tabular}{|c|c|c|c|}
\hline Gene & Function & Putative NMD-inducing feature & Relative increase (microarray value) \\
\hline Asparagine synthetase ( $A S N S$ ) & Asparagine biosynthesis & uORF & 4.7 \\
\hline Cystathionine-g-lyase $(\mathrm{CTH})$ & Cysteine biosynthesis & Unknown & 4.0 \\
\hline Pyrroline-5-carboxylate reductase 1 (PYCR 1) & Proline biosynthesis & Unknown & 2.0 \\
\hline Phosphoglycerate dehydrogenase $(P H G D H)$ & Serine biosynthesis & uORF & 2.0 \\
\hline Cysteinyl-tRNA synthetase (CARS) & tRNA charging & Unknown & 3.3 \\
\hline Seryl-tRNA synthetase (SARS) & tRNA charging & Unknown & 2.4 \\
\hline Leucyl-tRNA synthetase, mitochondrial ( $L A R S 2$ ) & tRNA charging & uORFs & 2.0 \\
\hline Nephropathic cystinosis (CTNS) & Amino acid transport & uORF & 2.2 \\
\hline Proton/amino acid symporter (SLC36A1) & Amino acid transport & uORF & 4.4 \\
\hline Glutamate/neutral amino acid transporter (SLC1A4) & Amino acid transport & Unknown & 4.2 \\
\hline Neutral amino acid transporter (SLC1A5) & Amino acid transport & Unknown & 2.2 \\
\hline Dibasic/neutral amino acid transporter (SLC3A2) & Amino acid transport & uORFs, Alt. exon with PTC & 2.7 \\
\hline Activating transcription factor 3 (ATF3) & Transcription factor & Alt. exon with PTC & 9.6 \\
\hline Activating transcription factor 4 (ATF4) & Transcription factor & uORFs & 4.1 \\
\hline
\end{tabular}

a distinct mechanism for recognizing nonsense codons that relies on splicing is used ${ }^{10}$. As yeast NMD occurs independently of splicing ${ }^{11}$, unique classes of endogenous NMD substrates probably exist in higher eukaryotes.

We used microarrays to examine transcript expression profiles after short interfering RNA (siRNA)-mediated depletion of Rent1 in HeLa cells $^{3}$. We expressed $\sim 4,000$ transcripts, representing $33 \%$ of the probe sets on the arrays, in HeLa cells and then assayed them in these experiments. We found that 197 transcripts (4.9\%) were consistently upregulated (Supplementary Table 1 online) and 176 transcripts $(4.4 \%)$ were consistently downregulated (Supplementary Table 2 online) by a factor of at least 1.9 in duplicate experiments.

An intron located at least 50-55 nucleotides downstream of a termination codon is sufficient to initiate mammalian $\mathrm{NMD}^{5}$. More than half of the upregulated transcripts (104 of 197) had identifiable features that satisfied this constraint (Table 1 and Supplementary (2) Table 1 online). Putative NMD-inducing features included upstream open reading frames (uORFs; 70 transcripts), alternative splicing that introduces nonsense codons or frameshifts (21 transcripts, some of which undergo alternative splicing specifically in HeLa cells ${ }^{12,13}$ ) and introns in the $3^{\prime}$ untranslated region (UTR; 9 transcripts). Two transcripts encoding proteins containing selenocysteine were also upregulated, consistent with the alternative recognition of the UGA selenocysteine codon as a signal for translation termination ${ }^{14}$. Additionally, we observed upregulation of nonfunctional transcripts derived from mariner 2 transposon (HSMAR2) remnants and human endogenous retrovirus $\mathrm{H}(H E R V-H)$ sequences that have acquired premature termination codons (PTCs) during evolution. Previous studies ${ }^{15-17}$ and our analyses of expressed-sequence tags showed that at least some HSMAR2 and HERV-H transcripts undergo splicing. Accordingly, we observed $\sim 1.0$-kb transcripts corresponding to the size of the spliced $H E R V-H$ message (Fig. 1c).

To confirm that transcripts that were upregulated in cells depleted of Rentl were primary substrates of the NMD pathway, we determined representative mRNA decay rates (Fig. 1a,b and Table 2). All 14 transcripts that we examined had prolonged half-lives in Rent1-depleted cells, consistent with direct regulation by nonsense surveillance. Reliable half-lives of transposon- or retrovirus-derived transcripts could not be measured as they are encoded by thousands of loci ${ }^{15,16}$ that cannot be individually discriminated by northern blotting and probably have widely variable decay rates. We observed increased steady-state abundance of these RNAs, validating the microarray

results (Fig. 1c,d). To confirm that the upregulated transcripts represent bona fide NMD substrates, we examined the steady-state abundance of a representative set in cells depleted of a second essential trans-effector of nonsense surveillance, Rent2 (also called hUpf2). We observed concordant upregulation of all five transcripts examined (Fig. 1d). We also observed increased expression of selected transcription factors (e.g., ATF3, ATF4, TCFL4 and CEBPG),

a

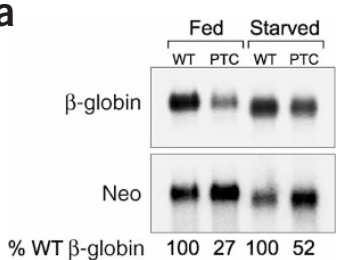

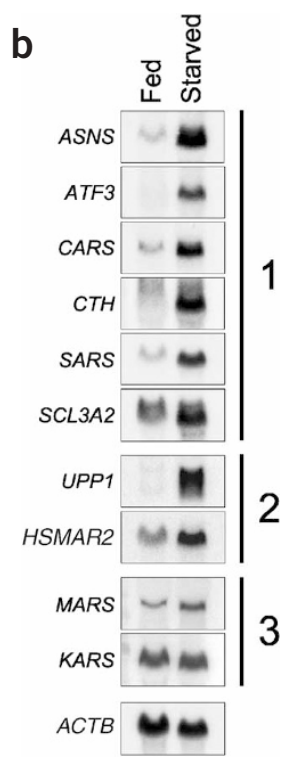

Figure 2 Amino acid starvation inhibits NMD and upregulates transcripts required for amino acid homeostasis. (a) Cells were transfected with a wildtype (WT) or nonsense-containing (PTC) $\beta$-globin minigene and grown in the presence (Fed) or absence (Starved) of amino acids. The steady-state abundance of $\beta$-globin messages was determined by northern blotting. Levels of neomycin phosphotransferase II ( $\mathrm{NeO}$ ), also encoded on the minigene plasmid, were used to control for differences in transfection efficiency and loading. (b) Northern-blot analysis of physiologic NMD substrates in the presence and absence of amino acids. The six transcripts in the upper portion of the figure (group 1) have functions related to amino acid homeostasis, whereas UPP1 and HSMAR2 (group 2) do not. MARS and $K A R S$ encode transcription factors related to amino acid metabolism but were not upregulated by NMD inhibition (group 3 ). $\beta$-actin serves as a loading control. 
Table 4 Starvation-response yeast transcripts upregulated upon NMD inhibition

\begin{tabular}{|c|c|c|}
\hline Category & Gene & Function \\
\hline \multirow[t]{10}{*}{ Amino acid biosynthesis/metabolism } & SER3 & Serine biosynthesis \\
\hline & $T R P 1$ & Tryptophan biosynthesis \\
\hline & CPA1 & Arginine biosynthesis \\
\hline & HIS3 & Histidine biosynthesis \\
\hline & ALD3 & Alanine biosynthesis \\
\hline & IDP2 & Glutamate biosynthesis \\
\hline & LEU3 & Leucine biosynthesis \\
\hline & HIS5 & Histidine biosynthesis \\
\hline & LYS5 & Lysine biosynthesis \\
\hline & GDH2 & Glutamate metabolism \\
\hline \multirow[t]{8}{*}{ Amino acid permeases or related functions } & CAN1 & Basic amino acid permease \\
\hline & AGP3 & Low-affinity amino acid permease; prominent in response to starvation \\
\hline & $A L P 1$ & Basic amino acid permease \\
\hline & ODC2 & Mitochondrial transport (lysine and glutamate biosynthesis) \\
\hline & $S S Y 1$ & Amino acid sensor; regulates amino acid permease expression \\
\hline & LST7 & Regulates transport of amino acid permeases from Golgi to cell surface \\
\hline & ARG81 & Arginine sensor; required for arginine-dependent activation of $C A R 1$ (arginase) \\
\hline & OPT2 & Oligopeptide transport \\
\hline \multirow[t]{4}{*}{ Autophagy } & ATG5 & Involved in autophagy \\
\hline & ATG12* & Essential for autophagy \\
\hline & ATG10 & Protein-vacuolar targeting; autophagy \\
\hline & ATG17 & Activation of $A P G 1$ protein kinase \\
\hline \multirow[t]{8}{*}{ Allantoate utilization } & $D A L 5^{*}$ & Allantoate permease \\
\hline & YIL166C & Similar to $D A L 5$ (function inferred) \\
\hline & YLL055W & Similar to $D A L 5$ (function inferred) \\
\hline & $D A L 2$ & Allantoicase \\
\hline & $D A L 7$ & Malate synthase (allantoin degradation) \\
\hline & $D A L 3$ & Ureidoglycolate (allantoin degradation) \\
\hline & DAL82 & Positive regulator of allophanate-inducible genes \\
\hline & DUR1,2 & Allophonate hydrolase (also urea carboxylase) \\
\hline \multirow[t]{3}{*}{ Urea and ammonia utilization } & DUR3 & Urea transporter \\
\hline & DUR1,2 & Urea carboxylase (also allophonate hydrolase) \\
\hline & MEP2 & Ammonia sensor and transporter \\
\hline \multirow[t]{10}{*}{ Sporulation } & SP016 & Required for spore formation \\
\hline & SPS4 & Sporulation-specific protein \\
\hline & YLL033W & Involved in sporulation \\
\hline & $T E P 1$ & Tyrosine phosphatase; spore-wall assembly \\
\hline & ISC10 & Required for sporulation \\
\hline & $R / M 21$ & Involved in sporulation \\
\hline & SPR6 & Involved in sporulation \\
\hline & $A D Y 3$ & Required for spore-wall formation \\
\hline & DON1 & Assembly of prospore membrane \\
\hline & ADY4 & Triggers formation of prospore membrane \\
\hline
\end{tabular}

\footnotetext{
*These genes have a Gcn4p-independent response to starvation.
}

indicating that there are probably also some indirect effects on transcript abundance.

In contrast to the upregulated transcripts, the seven downregulated transcripts that we tested either showed no change in steady-state abundance or stability on depletion of Rent1 or showed no concordant changes on depletion of Rent2, as assessed by northern blotting (data not shown). Thus, as in yeast ${ }^{8}$, we conclude that few, if any, mammalian transcripts that showed decreased abundance by microarray analysis are regulated by NMD.

As in yeast ${ }^{8}$, we observed upregulation of numerous transposonderived RNAs and transcripts containing uORFs in NMD-deficient mammalian cells, indicating that these are conserved classes of NMDregulated transcripts. The other classes of NMD substrates in yeast were not identified in our analyses (e.g., pseudogene and polycistronic transcripts; and transcripts with inefficient pre-mRNA splicing, leaky scanning or +1 frameshifting); this absence probably reflects the limited composition of current mammalian microarrays and incomplete annotation of the human genome.

The requirement for splicing in mammalian nonsense-codon recognition has given rise to new classes of NMD substrates, including alternatively spliced transcripts and transcripts with introns in their $3^{\prime}$ UTRs. Bioinformatic analyses have suggested that introduction of PTCs through alternative splicing is a relatively common feature of the human transcriptome ${ }^{18}$. When coupled with NMD, this provides a mechanism to achieve regulated titration of gene expression using the complex alternative splicing machinery, while avoiding the potentially deleterious consequences of expression of truncated proteins. 
Among physiologic NMD substrates, we observed increased representation of genes involved in amino acid metabolism (Table 3). Of the 80 genes on the array assigned to gene ontology terms 'amino acid transport', 'amino acid biosynthesis' and 'amino acid activation', 12 $(15 \%)$ were upregulated after abrogation of NMD. Given that only $4.9 \%$ of assayed transcripts were upregulated overall, this is a significant enrichment $\left(\chi^{2}=16.3, P<10^{-4}\right)$. We also observed upregulation of two transcription factors that coordinate cellular responses to amino acid starvation (ATF3 and ATF4; refs. 19,20). os Depletion of amino acids inhibits translation ${ }^{21}$, and as NMD requires ongoing translation, regulation of these transcripts by nonsense surveillance probably couples their expression level to translational efficiency. Thus, under conditions of amino acid starvation, inhibition of translation and NMD increases expression of transcripts that promote restoration of amino acid homeostasis. In keeping with this hypothesis, we observed attenuation of NMD under conditions of amino acid starvation, as assessed by the increased abundance of nonsense transcripts derived from a $\beta$-globin minigene and the increased abundance of representative NMD-regulated transcripts both with and without functions related to amino acid homeostasis (Fig. 2). As expected, some transcripts related to amino acid homeostasis that were not upregulated by NMD inhibition showed increased abundance in response to starvation (e.g., MARS), whereas 을 others did not (e.g., KARS). These data attest to the complexity of the response to nutrient deprivation. Our observations support the view that NMD contributes to the regulation of transcripts that encode key regulators of the starvation response. Moreover, there is adequate precedent that starvation-induced translational inhibition can be associated with an increased translational yield for selected transcripts, including ATF3 and ATF4 (refs. 19,22).

Notably, analysis of a previously determined set of yeast genes that have increased expression in response to NMD inhibition ${ }^{8}$ uncovered evidence that this mechanism to preserve amino acid homeostasis is evolutionarily conserved (Table 4). Upregulated transcripts include SSY1, encoding a sensor of external amino acid concentration that also coordinates transcription of amino acid permease genes in response to nutrient deprivation; LST7, encoding a positive regulator of transport of amino acid permeases from the Golgi to the cell surface; and AGP3,

encoding a low-affinity permease that might have a prominent role in amino acid transport induced by nitrogen starvation ${ }^{23,24}$. Other upregulated transcripts encode factors involved in the transport and catabolism of secondary nitrogen sources ${ }^{25}$, including the only known allantoate permeases, two putative allantoate permeases, five of six factors known to mediate allantoin degradation, a urea transport and degradation enzyme, and a sensor and transporter of ammonia. NMD inhibition was also associated with increased expression of crucial mediators of autophagy (bulk vacuolar degradation of cytosolic proteins to recycle amino acids in response to starvation) and sporulation. Multiple yeast transcripts that show increased expression after NMD inhibition (e.g., TRP1, ATG12 (also called APG12) and DAL5) have been shown experimentally to lack responsiveness to Gcn4p, a master transcriptional activator of amino acid biosynthetic genes in multiple pathways ${ }^{26}$, suggestive of complementarity between transcriptional and post-transcriptional responses to amino acid deprivation. Consistent with the paradigm that NMD has been functionally incorporated into homeostatic mechanisms, we also observed upregulation of the human homolog of Caenorhabditis elegans smg-5, a gene that is essential for mammalian $\mathrm{NMD}^{27}$, in NMD-deficient HeLa cells. This may be a mechanism by which NMD regulates its own efficiency.

Our results suggest that the predominant role of mammalian NMD is to regulate the expression of thousands of physiologic transcripts. In this view, its ability to modify disease caused by nonsense mutations, though medically important, is inconsequential for evolutionary selection. Given the number and diversity of transcripts regulated by NMD, the process has probably been functionally integrated into additional developmental and homeostatic mechanisms. This may explain the unique dependence of mammals on NMD for viability ${ }^{28}$ and limit therapeutic strategies based on modulation of the pathway. NMD also mutes a noisy genome and may thus provide tolerance for nonproductive events that occur through genome evolution. This may serve to buffer and hence maintain variation that proves beneficial only under selected physiologic or environmental conditions.

\section{METHODS}

Cell culture, siRNA treatment and RNA analysis. We cultured HeLa cells in Dulbecco's modified Eagle medium supplemented with $10 \%$ fetal bovine serum and carried out siRNA-mediated silencing of Rent 1 and Rent 2 as described ${ }^{3}$. We isolated RNA using Trizol (Invitrogen) $72 \mathrm{~h}$ after siRNA treatment and purified it using the RNeasy Mini Kit (Qiagen). As a positive control for the efficacy of siRNA treatment, we transfected an aliquot of the cells treated by RNA interference with a PTC-containing T-cell receptor $\beta$ minigene (a gift from M. Wilkinson, University of Texas, MD Anderson Cancer Center) as described $^{3}$ and determined the efficiency of NMD by northern blotting. We observed potent inhibition of NMD in the cells treated with siRNA (data not shown). Successful siRNA treatment was also indicated by decreased RENT1 mRNA levels, as assessed by microarray analysis (Supplementary Table 2 online). For mRNA half-life experiments, we treated cells with $100 \mu \mathrm{g} \mathrm{ml} \mathrm{of} \mathrm{5,6-}$ dichloro-1- $\beta$-D-ribofuranosylbenzimidazole and collected RNA. For amino acid starvation experiments, we starved cells for $20 \mathrm{~h}$ in Krebs-Ringer bicarbonate buffer supplemented with $10 \%$ dialyzed fetal bovine serum. We grew fed cells in parallel in Dulbecco's modified Eagle medium supplemented with $10 \%$ dialyzed fetal bovine serum. We obtained the $\beta$-globin minigene from J. Lykke-Andersen (University of Colorado) and J. Steitz (Yale University). We generated northern-blot probes by RT-PCR (primer sequences available on request) and carried out hybridizations with UltraHyb (Ambion). We quantified radioactive signals using an Instant Imager (Packard) and calculated halflives as described ${ }^{29}$.

Microarray hybridization and data analysis. We processed total RNA from control and experimental cell preparations (two independent replicates for each) using single-round RNA amplification protocols as described in the Affymetrix GeneChip Expression Analysis Technical Manual. We used the SuperScript Choice System (Invitrogen) to synthesize first-strand cDNA from $5 \mu \mathrm{g}$ of total RNA using a primer with oligo dT and T7 promoter sequences (Proligo LLC). After double-stranded cDNA synthesis, we purified the product by phenol-chloroform extraction and generated biotinylated antisense cRNA through in vitro transcription using the BioArray RNA High Yield Transcript Labelling kit (ENZO Life Sciences). After fragmenting cRNA at $94{ }^{\circ} \mathrm{C}$ for $35 \mathrm{~min}$ in $100 \mathrm{mM}$ Tris-acetate $(\mathrm{pH} 8.2), 500 \mathrm{mM}$ potassium acetate and $150 \mathrm{mM}$ magnesium acetate, we hybridized $10 \mu \mathrm{g}$ of cRNA to the Affymetrix human genome GeneChip array U95Av2 for $16 \mathrm{~h}$ at $45{ }^{\circ} \mathrm{C}$ with constant rotation. We used a Fluidics Station 400 (Affymetrix) to wash and stain the chips. Fluorescence was detected using a G2500 GeneArray Scanner (HewlettPackard) and image analysis of each GeneChip was done through the Microarray Suite 5.0 software from Affymetrix (MAS 5.0). For comparison between different chips, we used global scaling with a user-defined target intensity of 150 .

We carried out quality control analysis of RNA samples using the Agilent Bioanalyzer Lab on a Chip (Agilent Technologies), confirming that all samples had optimal rRNA ratios (1:2 for 18S:28S). For quality control of hybridizations, GeneChip images and comparisons between chips, we studied the following parameters: scaling factor (all resulting values were within comparable range), background (all values were between 36 and 48), percentage of present calls (all chips had $31-33 \%$ present), housekeeping genes ( $3^{\prime}: 5^{\prime}$ ratios of GAPD were consistently between 1.1 and 1.3 ) and presence or absence of internal spike controls (Bio B was present in all cases).

The initial analysis of the expression results was based on pairwise comparisons among the different experimental conditions. Any transcript whose 
expression level changed by a factor of at least 1.9 between experimental sample and control sample was considered to be significantly differentially expressed. We chose 1.9 as the cut-off value because at or above this value, transcripts showed full reproducibility in our replicate analyses. The results obtained from the duplicate samples were filtered independently for significance on each of the four iterative pairwise comparisons. We selected transcripts that were significant in at least two of the four comparisons for the final gene candidate list. The relative change values were converted from the average signal log ratio values obtained from MAS 5.0.

Analysis of genomic organization and alternative splicing of upregulated transcripts. We used publicly available databases, including the National Center for Biotechnology Information and the University of California Santa Cruz genome browser, to determine transcript structures.

Accession numbers. GenBank: spliced HSMAR2 expressed-sequence tags, T98067, T40030, R96154, U92014 and U92019. GEO: raw microarray data: luciferase control 1, GSM29530; luciferase control 2, GSM29531; RENT1-1, GSM29532; RENT1-2, GSM29534; series 'determination of mRNA transcripts in HeLa cells that are regulated by RENT1', GSE1703.

Note: Supplementary information is available on the Nature Genetics website.

\section{ACKNOWLEDGMENTS}

We thank D. Arking for assistance with statistical analyses; M. Awad and

C. ap Rhys for discussions; and M. Wilkinson, J. Lykke-Andersen, J. Steitz and

K. O'Donnell for reagents. Microarray hybridizations and data analysis were done

글 at the Johns Hopkins Medical Institutions Microarray Core Facility. H.C.D. is an

을 Investigator of the Howard Hughes Medical Institute.

\section{COMPETING INTERESTS STATEMENT}

The authors declare that they have no competing financial interests.

Received 22 June; accepted 20 August 2004

Published online at http://www.nature.com/naturegenetics/

1. Wagner, E. \& Lykke-Andersen, J. mRNA surveillance: the perfect persist. J. Cell Sci. 115, 3033-3038 (2002).

2. Hentze, M.W. \& Kulozik, A.E. A perfect message: RNA surveillance and nonsensemediated decay. Cell 96, 307-310 (1999).

3. Mendell, J.T., ap Rhys, C.M. \& Dietz, H.C. Separable roles for rent1/hUpf1 in altered splicing and decay of nonsense transcripts. Science 298, 419-422 (2002).

4. Sun, X., Perlick, H.A., Dietz, H.C. \& Maquat, L.E. A mutated human homologue to yeast Upf1 protein has a dominant-negative effect on the decay of nonsense-containing mRNAs in mammalian cells. Proc. Natl. Acad. Sci. USA 95, 10009-10014 (1998).

. Nagy, E. \& Maquat, L.E. A rule for termination-codon position within intron-containing genes: when nonsense affects RNA abundance. Trends Biochem. Sci. 23, 198-199 (1998).

6. Inoue, K. et al. Molecular mechanism for distinct neurological phenotypes conveyed by allelic truncating mutations. Nat. Genet. 36, 361-369 (2004).

7. Frischmeyer, P.A. \& Dietz, H.C. Nonsense-mediated mRNA decay in health and disease. Hum. Mol. Genet. 8, 1893-1900 (1999).

8. He, F. et al. Genome-wide analysis of mRNAs regulated by the nonsense-mediated and $5^{\prime}$ to $3^{\prime}$ mRNA decay pathways in yeast. Mol. Cell 12, 1439-1452 (2003).

9. Lelivelt, M.J. \& Culbertson, M.R. Yeast Upf proteins required for RNA surveillance affect global expression of the yeast transcriptome. Mol. Cell. Biol. 19, 6710-6719 (1999).
10. Zhang, J., Sun, X., Qian, Y. \& Maquat, L.E. Intron function in the nonsense-mediated decay of beta-globin mRNA: indications that pre-mRNA splicing in the nucleus can influence mRNA translation in the cytoplasm. RNA 4, 801-815 (1998).

11. Zhang, S., Ruiz-Echevarria, M.J., Quan, Y. \& Peltz, S.W. Identification and characterization of a sequence motif involved in nonsense-mediated mRNA decay. Mol. Cell. Biol. 15, 2231-2244 (1995).

12. Chen, B.P., Liang, G., Whelan, J. \& Hai, T. ATF3 and ATF3 delta Zip. Transcriptional repression versus activation by alternatively spliced isoforms. J. Biol. Chem. 269, 15819-15826 (1994).

13. Aubel, C., Chabanon, H., Carraro, V., Wallace, H.M. \& Brachet, P. Expression of spermidine/spermine N1-acetyltransferase in HeLa cells is regulated by amino acid sufficiency. Int. J. Biochem. Cell. Biol. 35, 1388-1398 (2003).

14. Moriarty, P.M., Reddy, C.C. \& Maquat, L.E. Selenium deficiency reduces the abundance of mRNA for Se-dependent glutathione peroxidase 1 by a UGA-dependent mechanism likely to be nonsense codon-mediated decay of cytoplasmic mRNA. Mol. Cell. Biol. 18, 2932-2939 (1998).

15. Robertson, H.M. \& Martos, R. Molecular evolution of the second ancient human mariner transposon, Hsmar2, illustrates patterns of neutral evolution in the human genome lineage. Gene 205, 219-228 (1997).

16. Goodchild, N.L., Freeman, J.D. \& Mager, D.L. Spliced HERV-H endogenous retroviral sequences in human genomic DNA: evidence for amplification via retrotransposition. Virology 206, 164-173 (1995).

17. Lindeskog, M. \& Blomberg, J. Spliced human endogenous retroviral HERV-H env transcripts in T-cell leukaemia cell lines and normal leukocytes: alternative splicing pattern of HERV-H transcripts. J. Gen. Virol. 78, 2575-2585 (1997).

18. Lewis, B.P., Green, R.E. \& Brenner, S.E. Evidence for the widespread coupling of alternative splicing and nonsense-mediated mRNA decay in humans. Proc. Natl. Acad. Sci. USA 100, 189-192 (2003).

19. Pan, Y., Chen, H., Siu, F. \& Kilberg, M.S. Amino acid deprivation and endoplasmic reticulum stress induce expression of multiple activating transcription factor-3 mRNA species that, when overexpressed in HepG2 cells, modulate transcription by the human asparagine synthetase promoter. J. Biol. Chem. 278, 38402-38412 (2003).

20. Siu, F., Bain, P.J., LeBlanc-Chaffin, R., Chen, H. \& Kilberg, M.S. ATF4 is a mediator of the nutrient-sensing response pathway that activates the human asparagine synthetase gene. J. Biol. Chem. 277, 24120-24127 (2002).

21. Pain, V.M. Translational control during amino acid starvation. Biochimie 76, 718-728 (1994).

22. Averous, J. et al. Induction of $\mathrm{CHOP}$ expression by amino acid limitation requires both ATF4 expression and ATF2 phosphorylation. J. Biol. Chem. 279, 5288-5297 (2004).

23. Forsberg, H. \& Ljungdahl, P.O. Sensors of extracellular nutrients in Saccharomyces cerevisiae. Curr. Genet. 40, 91-109 (2001).

24. Schreve, J.L. \& Garrett, J.M. Yeast Agp2p and Agp3p function as amino acid permeases in poor nutrient conditions. Biochem. Biophys. Res. Commun. 313, 745-751 (2004).

25. Hardwick, J.S., Kuruvilla, F.G., Tong, J.K., Shamji, A.F. \& Schreiber, S.L. Rapamycinmodulated transcription defines the subset of nutrient-sensitive signaling pathways directly controlled by the Tor proteins. Proc. Natl. Acad. Sci. USA 96, 14866-14870 (1999).

26. Natarajan, K. et al. Transcriptional profiling shows that Gcn4p is a master regulator of gene expression during amino acid starvation in yeast. Mol. Cell. Biol. 21, 4347-4368 (2001).

27. Gatfield, D., Unterholzner, L., Ciccarelli, F.D., Bork, P. \& Izaurralde, E. Nonsensemediated mRNA decay in Drosophila: at the intersection of the yeast and mammalian pathways. EMBO J. 22, 3960-3970 (2003).

28. Medghalchi, S.M. et al. Rent1, a trans-effector of nonsense-mediated mRNA decay, is essential for mammalian embryonic viability. Hum. Mol. Genet. 10, 99-105 (2001).

29. Leeds, P., Peltz, S.W., Jacobson, A. \& Culbertson, M.R. The product of the yeast UPF1 gene is required for rapid turnover of mRNAs containing a premature translational termination codon. Genes Dev. 5, 2303-2314 (1991).

30. Leeds, P., Wood, J.M., Lee, B.S. \& Culbertson, M.R. Gene products that promote mRNA turnover in Saccharomyces cerevisiae. Mol. Cell. Biol. 12, 2165-2177 (1992). 